\title{
Contents, Vol. 114, 1947
}

\section{Index}

Amsler, M., L'Examen qualitalif de la function maculaire .... 248

Discussion: Fischer, Goldmann. Schlußwort: Amsl·er. Appelmans, M., La kéraíite ponctuée superficielle chro $\pi$ ique par onchocerca volvulus 129

Babel, J., Myosites orbitaires chroniques - L'importance des lesions veineuses de Torbite 312

Bamatter, F., La choriorétinite toxoplasmique (avec planche V) . . 340 Bangertcr, A., Zur

Behandlung bei Tränenröhrchenstenose .... 195

Diskussion: Knapp.

- Zur Behandlung des Ektropiums 203

Diskussion: Knapp.

Bíschler, V., Un cas de kysle congenital scléro-cornéen (avec planche VI) 371 Boslröm, C. G., und Kugelberg, I., Die amlliche Farbensinnkontrolle in

Schweden 95

Bourquin,J.B., Eosinophilie conjoncfivale et eosinophilie sanguine (avec

planche 1) 203

Discussion: Chomé. Bürki, E., Über ein neues Veri'ahren zur Konservierung von Horahautgewebs 283

Diskussion: Kenel, Bürki. Danis, P., vide Maeder, G. Diethelm, W., Über Ectopia lentis ohn·e Arachnodaktylie und ihre Bezie-

hungen zur Ecíopia lentis et pupillae 16

Dufour, R., Méthodes modernes de repérage des corps étrangers oculaires 298

Discussion: Goldmann.Fischer, F. P., Über Linsenstoffwechsel und Kataraktentstehung ...1 - $\quad$ und H. G. Moorrees, «Camp eyes» und Sonnenblendung .... 10Franceschetti, A., Rubéole pendant la grosscsse et cataracte congénitale

chez Гenfant, accompagnée du phénomène digito-oculaire . . 332 Discussion: Gjissing. Gút, L., Ein Beitrag zur Topographie des Ansatzes der vier geraden

Augenmuskeln

Ein interessanter Fall von Membrana pupillaris persevera $\pi$ s . . 52

Über die Wirksamkeit der modifizierten Cyclodialyse .... 106

On Partial Plasty of the Socket V 414

Goldmann, H., Studien über den Abflußdruck des Kammerwassers beirn

Menschen 81, 216

Diskussion: A. Bruckner, Fjscher. Schlußwort: Goldmann.

- $\quad$ Beilrag zur Angioskotometrie 147, 277

Diskussion: Fischer.

Hamburg, J., Die Steigerung der Zelloxydation als therapeutisches Prin- 
zip bei der Intoxikations-Amblyopie 358

Diskussion: Huber, Weber, Fischer.

Huber, A., Zur Pharmakologie der Blut-Kammenvasser-Schvanke . . 217 Diskussion: Goldmann, Fischer. Schlußwort: Huber.

- Zum Tyndall-Phänomen in der Augenvordierkammer .... 228Diskussion: Goldmann.

Huber, O., Ganzheitliches Denken und Augenheilkunde ... — 368

Diskussion: Amsler.Jonkers, G. H., On Objective Adaptometry (With plate VII) .... 397Knüsel, O., Über Sitz und Optik der Kontaktschalen 331

Diskussion: Dufour. Kugelberg, I., vide Boström, C. G.

Kuyz, 0., Zu Differential-diagnose von Maculaaffektionen (hierzu Taf. IV) 262 Maeder, G., et

Danis, P., Su $\gamma$ une nouvelle forme de dystrophie corné-enne (dystrophia filiformis profunda

corneae) associée à un ké.rato-

cone (avec planche III) 246

Discussion: Amsler.

Matteucci, P., Sur la nature de Tinnervation du muscle ciliaire de

Brücke-IVallace de Гhomme 377

Mihályhegyi, G., Neue Erfahruสgen mit der Anwendung von Kontakt-

schal-en ‘ 159

Moorrees, G., vide Fischer, F. P.

Much, V., Augensymptome nach Amöben-Dysenterie und bei Amöben-

tГägern $\quad 384$

No $\Gamma$ dmann, Jean, et Stoll, Roland, Contribution à $\Gamma$ étud $3 / 4$ des cellules

pigmentaires de la choroïde chez Гembryon de Poulet .... 99

Radnót, M., Über die Wirkung der Testosteronpräparate auf den intra-

ocularen Druck 168

- $\quad$ Über das flache Sarkom der Ade Thaut

409

Rintelen, F., Zur Kenntnis der Blickparesen 325

Diskussion: Goldmann, Verrey, Huber. Schlußwort: Rintelen. Safar, Karl, Iridodialyse nach

Starextraktion, geheilt durch Elektro-

koagulation mit dem Hochfrequenzstrom (Diathernie) ... 77 Sedan, Jean, et Sédan-Bauby,

Simone, La microponction des cataractes

hypermûres, insaisissables à la pince 65

Sédan-Bauby, S., vide Sedan, J.

Semadeni, E., Augenverletzungen durch Skispitze 304

Stern, H. J., Acute Ocular Manifestations of Ariboflavinosis . . 103 Stoll, R., vide Nordmann, J.

Streiff, E. B., Quelques résultats d'opérations plastiques .... 294 Verrey, F., Dégénérescence

pigmentaire de la rétine en secteurs symétri-

ques 278

- $\quad$ Myotonie dystrophique (maladie de Steinert) avec alteration maculairedegenerative

281

- «Kératoglobe aigu» 284

Vogelsanger, W., Ein Fall von Arachnoiditis optico-chiasmatica . 273 Weber, E., Das

symptomlose Netzhautloch in der augenärztlichen Praxis

(Hierzu Tafel II) 304

Diskussion: Amsler. 
- $\quad$ Mit Diplopie einhergehendes Konvergenzschielen Myoper (Biel-schowsky) 320

Weidmann, W., Über die Abbildungsschärfe des Auges

Zwahlen, P., Le priscol dans les brûlures oculaires 241

Discussion: Amsler.

GESELLSCHAFTSBERICHTE - SOCIETY TRANSACTIONS - SOCIÉTÉS

Groupe ophtalmologique du Léman. Seances des 6 et 7 avril 1946 à

Geneve 56

Vereinigung der Basler Augenärzte. Basel, den 22. Januar 1947 . 119

II. Pan American Congress of Ophthalmology 172, 418

Schweizerische Ophthalmologische Gesellschaft. 39. Generalversammlung in Bern, 21. und 22.

September 1946. Société Suisse d'Ophtalmologie. 39e Assemblée générale à Berne, 21 et 22

septembre $1946 \ldots 193$

B. BUCHBESPRECHUNGEN - BOOKS REVIEW - LIVRES NOUVEAUX

64

128

190 